\title{
Flow structures in end-view plane of slender delta wing
}

\author{
Besir Sahin ${ }^{1 *}$, Mehmet Oguz Tasci ${ }^{1}$, Ilyas Karasu ${ }^{2}$ and Huseyin Akilli ${ }^{1}$ \\ ${ }^{1}$ Department of Mechanical Engineering, Faculty of Engineering and Architecture, Cukurova University, 01330, Adana/ Turkey \\ ${ }^{2}$ Departments of Aerospace Engineering, Faculty of Aeronautics and Aerospace, Gaziantep University, 27310, Gaziantep/Turkey
}

\begin{abstract}
Present investigation focuses on unsteady flow structures in end-view planes at the trailing edge of delta wing, $\mathrm{X} / \mathrm{C}=1.0$, where consequences of vortex bursting and stall phenomena vary according to angles of attack over the range of $25^{\circ} \leq \alpha \leq 35^{\circ}$ and yaw angles, $\beta$ over the range of $0^{\circ} \leq \beta \leq 20^{\circ}$. Basic features of counter rotating vortices in end-view planes of delta win with $70^{\circ}$ sweep angle, $\Lambda$ are examined both qualitatively and quantitatively using Rhodamine dye and the PIV system. In the light of present experiments it is seen that with increasing yaw angle, $\beta$ symmetrical flow structure is disrupted continuously. Dispersed wind-ward side leading edge vortices cover a large part of flow domain, on the other hand, lee-ward side leading edge vortices cover only a small portion of flow domain.
\end{abstract}

\section{Introduction}

There are several factors that influence delta wing aerodynamics, particularly formations of vortical flow over delta wing. For example, primary factors are angle of attack $\alpha$, yaw angle $\beta$, sweep angle $\Lambda$, roll angle, $\theta$ wing thickness, t leading edge geometry and conditions of free-stream as well as Reynolds Number, Re. More precisely the geometry of delta wing has close relations with the formations of leading edge vortices and their bursting incidences. Lambourne and Bryer (1961) and Sarpkaya (1971) visualized a couple of vortex bursting events over a delta wing qualitatively which were called spiral and bubble type vortex bursting as shown in Figure 1.



Fig. 1. Types of vortex bursting in a tube (Sarpkaya, 1971)

Vortex bursting phenomena is key source of striking delta wing which triggers large mechanical vibrations leading to heavy fatigue destruction and causes the loss of load-bearing ability of a material under periodic load application.

Delta wings are differed from the other type of wings because a couple of leading edge vortices, separations, vortex breakdowns and chaotic vortical flows are generally available. Representation of flow field transformations over a delta wing at a certain angle of attack, $\alpha$ is given in Figure 2 (Anderson, 2001).

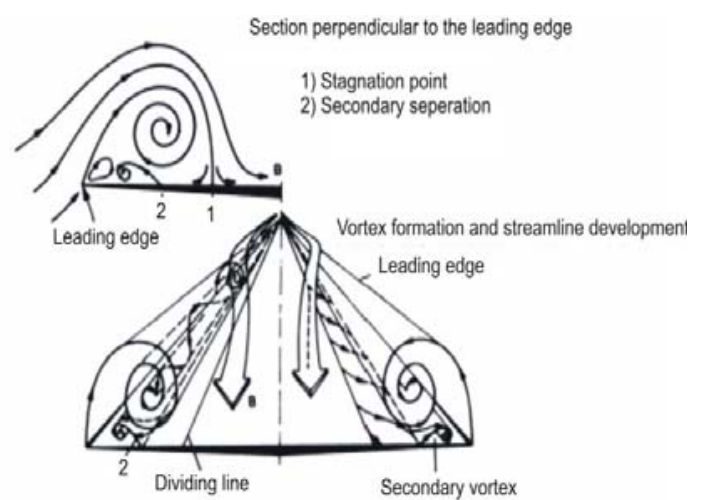

Fig. 2. Representation of flow field transformations over a delta wing under angle of attack, $\alpha$ (Anderson, 2001)

It is identified that aerodynamics of high angle of attack, $\alpha$ is one of the most important elements in aircraft design for the view of lift force $F_{L}$. A delta wing would also ensure high lift coefficient, $\mathrm{C}_{\mathrm{L}}$ at a larger angle of attack, $\alpha$ comparing to the wing of passenger aircrafts. Leading edge vortices at high angles of attack, $\alpha$ create most portion of lift forces, $\mathrm{F}_{\mathrm{L}}$. But, vortex bursting close to the surface of delta wing deteriorates maneuverability of air vehicles and causes material fatigues. Comprehension of these types of flows is very important for better aircraft maneuverability. A delta wing furnishes the Unmanned Combat Air Vehicles (UCAVs) with capability of sharp maneuvers and tactical advantages. Maneuverability of all these geometrical styles are restricted by the occurrence of vortex bursting and stall influencing both overall forces and moments of aircrafts.

Ozgoren et al. (2002) reported that there are five distinct layers of vorticity in the absence of vortex breakdown at low angle of attack, $\alpha$. Their delta wing

*Corresponding author: bsahin@cu.edu.tr

(C) The Authors, published by EDP Sciences. This is an open access article distributed under the terms of the Creative Commons Attribution License 4.0 (http://creativecommons.org/licenses/by/4.0/). 
model was highly swept which had $\Lambda=75^{\circ}$ and they monitored flow structure at attack angles of attack of $\alpha=24^{\circ}, 30^{\circ}, 32^{\circ}$ and $35^{\circ}$. Co-existing distributions of azimuthal vorticity were classified.

Akilli et al. (2001) conducted an experimental examination to search vortex bursting sensitiveness over a delta wing at high angles of attack $\alpha$, via placing very thin wire orthogonally to the center of leading-edge vortex. It is shown that a thin wire could move the onset of vortex bursting by as much as fifteen vortex diameters in the upstream direction.

Cross-flow studies in end-view planes of delta wings have not been conducted in detail. There are only a few studies available. For example, Yaniktepe and Rockwell (2005) performed experimental investigations on non-slender diamond and lambda type wings to analyze flow structures at the trailing edge zones. In both wings, vortical flow structures in the cross-flow planes of trailing edge change rapidly with angles of attack, $\alpha$ when delta wings have high sweep angle, $\Lambda$ more than 40 degree.

Although substantial scientific and technological information was obtained about instantaneous and timeaveraged flow structures of delta wings with relatively large sweep angles, $\Lambda$ in recent years, but influences of yaw angle, $\beta$ were not studied at all. Recent demands on UCAVs have encouraged researchers to pay more attentions in characterizing flow structures of delta wings. Present investigation focuses on the unsteady flow structure which occurs downstream of onset of vortex breakdown and services as a source of buffeting and stall that reduces the lift force, $\mathrm{F}_{\mathrm{L}}$.

Emphasizes in current investigation is given on the cross-flow in end-view planes at the trailing edge of delta wing, $\mathrm{X} / \mathrm{C}=1.0$ where consequences of vortex bursting and stall phenomena vary according to angles of attack, $\alpha$ and yaw angle, $\beta$. In the past, a great deal of effort was spent on the studies of delta wing aerodynamics with a high sweep angle $\Lambda$. It is possible to say that flow structure over this kind of wings is understood very well. But, research work in the case of low and moderate sweep angles, $\Lambda$ of delta wing are quite limited comparing to the delta wings with high sweep angles, $\Lambda$. On the other hand, it is worth to emphasize that the effect of yaw angles, $\beta$ on a delta wing aerodynamics have not been studied in detail yet. Particularly, there is no published work on the aerodynamics of slender delta wings as a function of yaw angle, $\beta$. For this reason, as a motivation of present work, further studies are required in order to understand flow structures and aerodynamics of slender delta wings in detail. A low and moderate sweep angled, $\Lambda$ delta wings, which are generic plan-forms of MAVs and UCAVs, have serious control and instability problems due to not having conventional aerodynamic control surfaces. For these wings; coactions between the leading edge vortex and boundary layer, the leading edge vortex bursting, a localized surface flow separation, effects of these incidents on a wing surface vibration and buffeting are among fundamental research topics. It is possible to discover a method to control instability problems by making further investigations on these topics and to understand flow phenomena better. Locations of vortex bursting over delta wings is not stable and displays fluctuation along the vortex axis.

Overall scene of vortex bursting close to the delta wing surface is presented in Figure 3 (Karasu et al., 2015). As seen in the figure, yaw angle, $\beta$ influences onset of vortex location in dramatically.
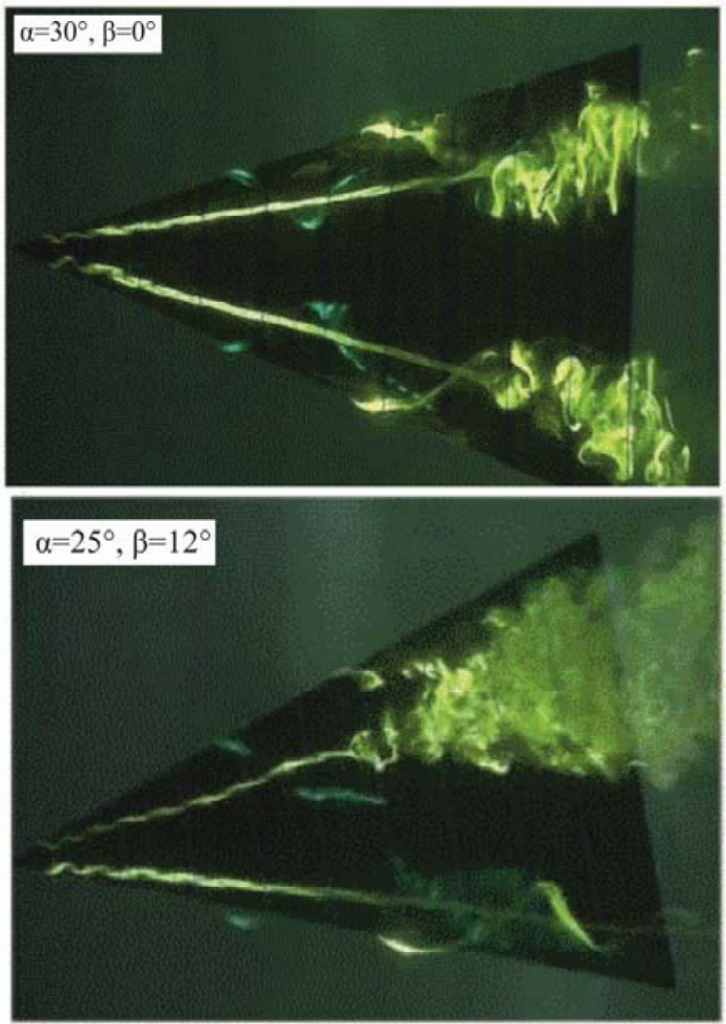

Fig. 3. Representation of symmetrical vortex bursting over a high sweep angle of delta wing, $\Lambda$ (Karasu et al., 2015)

Flow structures over the surface of non-slender diamond wing were studied by Yayla et al. (2009) using dye visualization and the Stereo Particle Image Velocimetry (PIV) System. In their study flow structures, turbulence statistics and vortex bursting transformation were examined by changing the data of yaw angle, $\beta$ within the range of $0^{\circ} \leq \beta \leq 15^{\circ}$ for attack angle of $\alpha=7^{\circ}$ at $\operatorname{Re}=1 \times 10^{4}$. They stated that when yaw angle, $\beta$ increases, the vortex bursting location on one side approaches to the wing apex, on the opposite side moves towards the trailing edge. Canpolat et al. (2009) monitored changes of flow structures occurred over a delta wing with respect to the angle of attack, $\alpha$ and yaw angle, $\beta$ having sweep angle, $\Lambda$ of delta wing as $40^{\circ}$. Flow characteristics over a delta wing in plan-view plane was reported for angles of attack, $\alpha=7^{\circ}, 10^{\circ}, 13^{\circ}$, and $17^{\circ}$, and yaw angles, $\beta=0^{\circ}, 6^{\circ}, 8^{\circ}$, and $15^{\circ}$. Dye visualizations of flow in cross flow planes were also reported at locations of $\mathrm{X} / \mathrm{C}=0.6,0.8$, and 1 . 0 .

Sohn et al. (2004) performed an experimental study on a yawed delta wing with inclusion of LEX employing dye visualizations. Interactions between Leading Edge Extension (LEX) and delta wing were investigated at some attack angles, $\alpha$ and yaw angles, $\beta$. They concluded 
that wing vortex and LEX vortex are coated around each other to have combined vortex which are comparably strengthened with a new identity when yaw angle, $\beta$ is taken as $0^{\circ}$. Visualization of vorticity consecrations over a double delta wing with and without yaw angle, $\beta$ was also reported by Sohn et. al, 2004. Experimental work on structures of flow over surface of a non-slender delta wing with $40^{\circ}$ sweep angle, $\Lambda$ was conducted by Canpolat et al. (2009). They stated that when a delta wing has certain value of a yaw angle $\beta$, symmetrical flow structures disappear and vortex bursting goes on earlier at the windward side with respect to the leeward side. They also discovered that main vortices in cross flow planes occurred inside of vortices close to central axis of delta wing. Yayla et al. (2010) performed experimental studies on an aerodynamics of a nonslender diamond wing which has $40^{\circ}$ sweep angle, $\Lambda$. They pointed out that yaw angle $\beta$ has substantial effects on the vortex bursting using the dye visualization technique. It was concluded that up to $4^{\circ}$ yaw angle, $\beta$ there are no clear changes in the vortex bursting locations, but at a higher yaw angle, $\beta$ after $4^{\circ}$, the point of vortex bursting moves towards the leading edge on the windward side, while this location moves further downstream on the leeward side. Locations of asymmetrical vortex bursting are seen over a delta wing in plain-view plane. Sohn and Chang (2010) observed the effect of central body on a yawed double delta wing using flow visualizations over a surface and wingsurface pressure measurements. They confirmed that up to $24^{\circ}$ attack angle, $\alpha$ availability of central body has a small effectiveness on the pressure distribution of suction side on the upper surface of wing, even at a large yaw angle of $\beta=20^{\circ}$.

\section{Material and Method}

Experiments were made in a close -loop open water channel in Fluid Mechanics Laboratory at Çukurova University. Dimensions of water channel are set as 8000 $\mathrm{mm}$ length, $1000 \mathrm{~mm}$ width, $750 \mathrm{~mm}$ height and test section of water tunnel is made by a transparent Plexiglas which has thickness of $15 \mathrm{~mm}$. The free-stream velocity is $120 \mathrm{~mm} / \mathrm{s}$ which corresponds to the Reynolds number of $\mathrm{Re}=2.10^{4}$ based on the cord length, $\mathrm{C}$ which is $120 \mathrm{~mm}$. The Delta wing was kept stationary in the test section by a special mechanism. The Delta wing was hold in horizontal position by a slender support arm that stretched from mid-chord of delta wing vertically. A few dye visualization experiments were conducted in order to have an idea, for example, how flow patterns behave in end-view planes of the delta wing at a location, $\mathrm{x} / \mathrm{c}=1.0$. Fluorescent rhodamine dyes which reflect laser light were used. Rhodamine dye was conveyed in a thin slot located along the surface of delta wing. SONY HDSR1 model video camera was employed. Schematic view of delta wing, measuring cross-section and experimental setup are shown in Fig. 4.

A 2D Particle Image Velocimetry (PIV) was used for capturing images of velocity fields. The PIV technique captures a general view of instantaneous flow data in a specified flow field. This feature lets user to examine even existence of small eddies with their effects and to provide opportunity for computing vorticity fields quantitatively. Particle Image Velocimetry (PIV) records the distance that particles move in time between two pulses of laser illuminations.

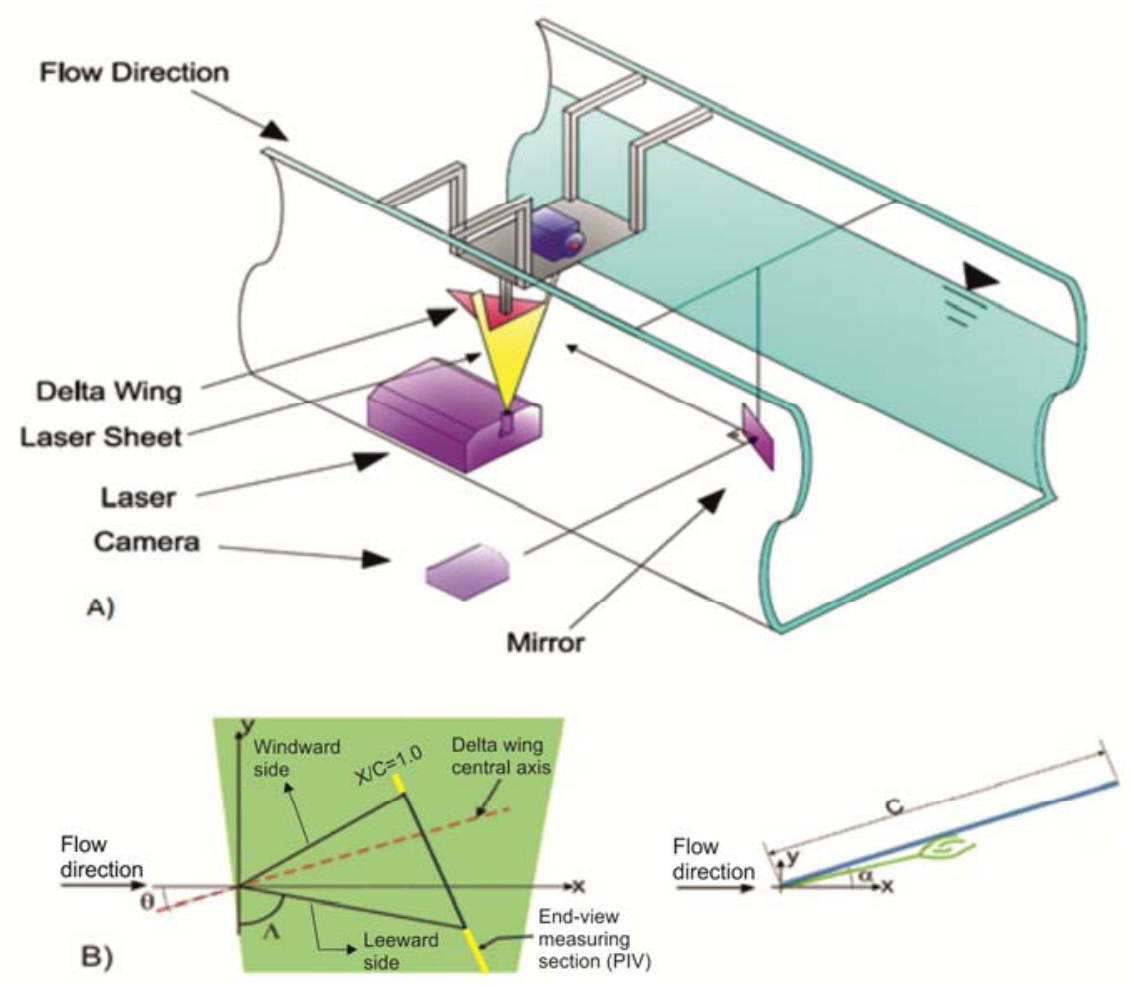

Fig. 4. A) Experimental Set-up of the PIV system, B) Schematic representation of the delta wing 
In general, the user can obtain image acquisition and data processing from the PIV system combined with the software and computer. Seeding particles moving in water flow were flashed by pulsating laser beams. A successive light sheet pulses provided the opportunity for the camera to construct an image map. Images were received by the $\mathrm{CCD}$ camera which has resolution of $1600 \times 1200$ pixels at a rate of 15 frames per second. Time delay between frames was taken as $170 \mu \mathrm{s}$. To eliminate bad vectors the CLEANVEC software was used (Meinhart and Soloff (1999)) in order to remove unwanted vectors. After that to fill the places of deleted vectors bilinear interpolation with least squares fit is used. During each test, a total 1050 images were captured. The laser light sheet was set normal to the flow direction. Mirror which had $600 \mathrm{~mm}-700 \mathrm{~mm}$ surface area located further downstream of the delta wing and was turned by $45^{\circ}$ from the free-stream flow direction in order to deliver the vision of delta wing and cross-flow domain to the camera.

\section{Result and Discussion}

The dye visualization experiments were firstly conducted in order to study vortical flow structures further downstream of the vortex bursting at a cross-flow plane, $\mathrm{X} / \mathrm{C}=1.0$. As soon as the leading edge vortices break down, a complex flow structure is developed. A pair of main counter rotating vortices take place in end-view planes at $\mathrm{X} / \mathrm{C}=1.0$. Figs. 5, 6 and 7 demonstrate that as soon as the location of vortex breakdown comes closer to the present measuring plane, $\mathrm{X} / \mathrm{C}=1.0$ the magnitude of interactions increases violently.

A small size vorticity concentrations occurred in the counter rotating main vorticities are also welldefined. Under high angles of attach, $\alpha$ the vortex break down occurs at an earlier stage of $\mathrm{X} / \mathrm{C}$. In this case, magnitude of large and small scales of vortices attenuated at $\mathrm{X} / \mathrm{C}=1.0$. As reported in the review work of the present study, Yaw angle, $\beta$ is very effective parameter in deteriorating the symmetrical flow structures leading to asymmetrical flow characteristics. Namely, on the leeward side ( Left hand side) of the delta wind, the locations of vortex break down moves further downstream while the oppositely oriented leading edge vortices breakdowns earlier. Either the leeward side or the windward side (Right hand side) leading edge vortex which ever breakdown close to end-view planes of the delta wing the magnitude of newly forming vortices become stronger. Here, free-stream flow direction is taken as a reference line for yaw angle, $\beta$ and locations of End-view plane or cross flow measuring plane, $\mathrm{X} / \mathrm{C}=1$ is fixed according to zero yaw angle, $\beta . \mathrm{A}$ quantitative visualization of vortical flow provides detailed physics of flow structures. In order to see the effect of yaw angle, $\beta$ on the flow structures in end-view plane of the delta wing time-averaged velocity vectors, $\langle\mathrm{V}\rangle$, streamline $\langle\Psi\rangle$ and vorticity, $\langle\omega\rangle$ are defined using instantaneous velocity data. As seen in figures, laser sheet is passed through the cross-section at
$\mathrm{X} / \mathrm{C}=1.0$ because dye visualization tests were conducted on the same cross-section.
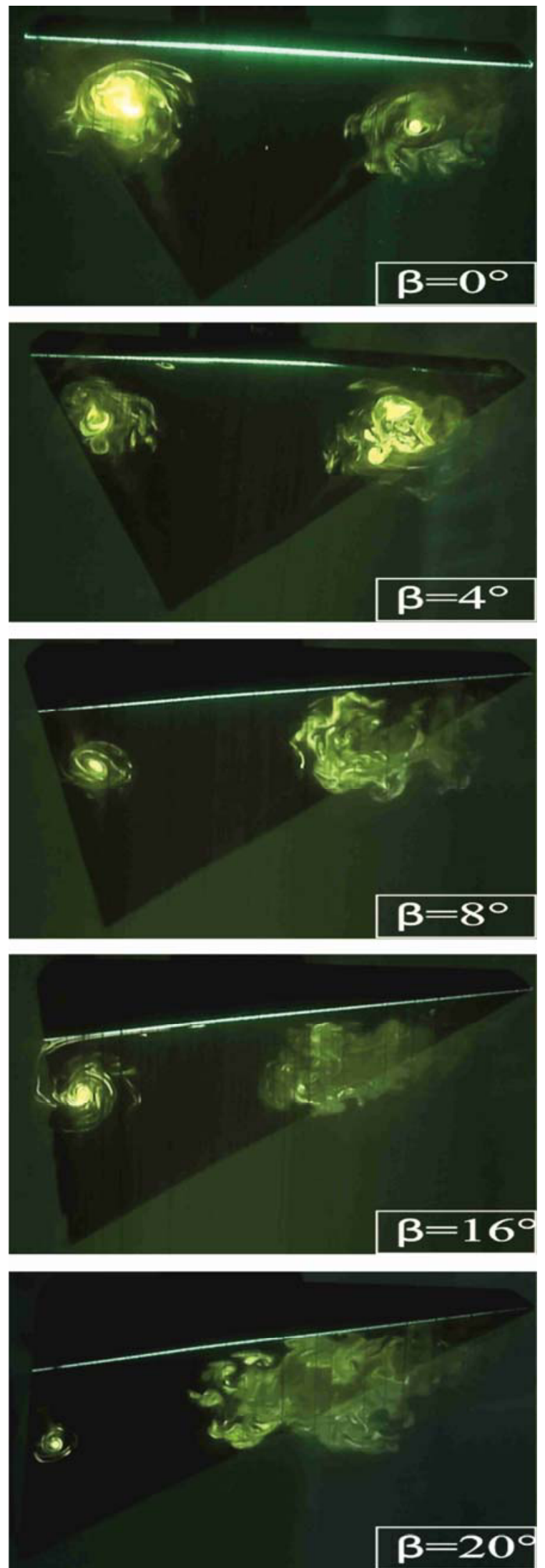

Fig. 5. Vortex formation in end-view plane as a function of yaw angle, $\beta$ at angle of attack, $\alpha=25^{\circ}$. The laser sheet is located at $\mathrm{x} / \mathrm{c}=1.0$ 

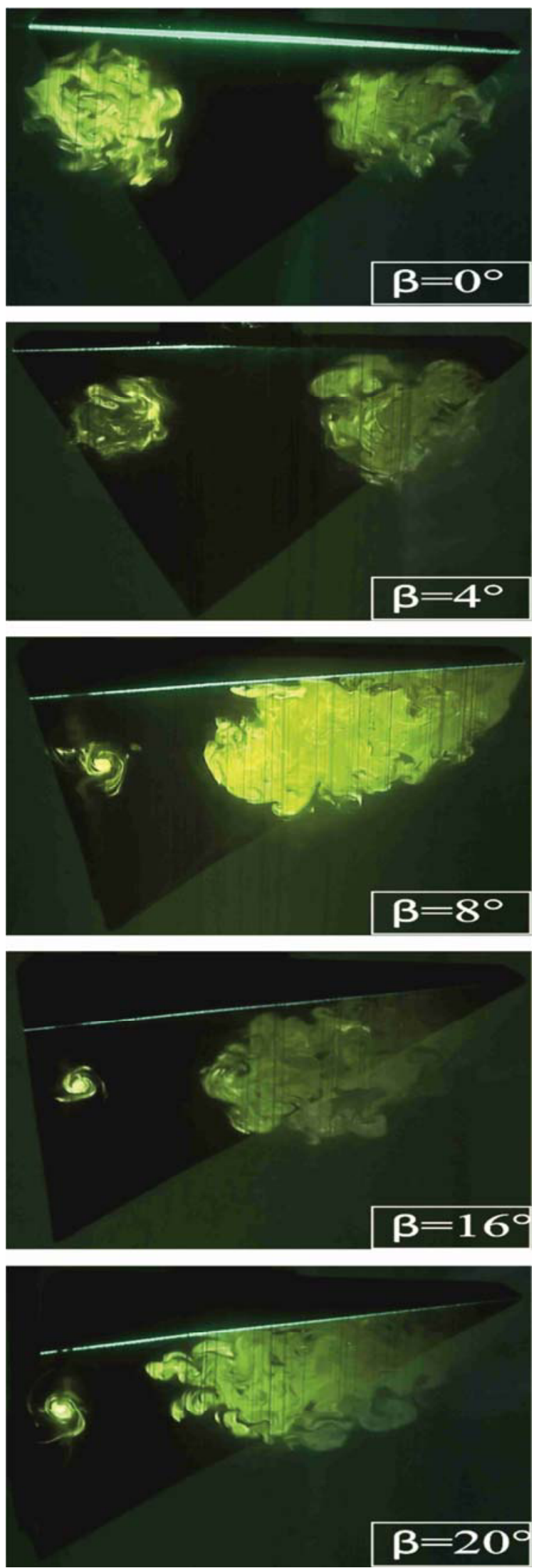

Fig. 6. Vortex formation in end-view plane as a function of yaw angle, $\beta$ at angle of attack, $\alpha=30^{\circ}$. The laser sheet is located at $\mathrm{X} / \mathrm{c}=1.0$
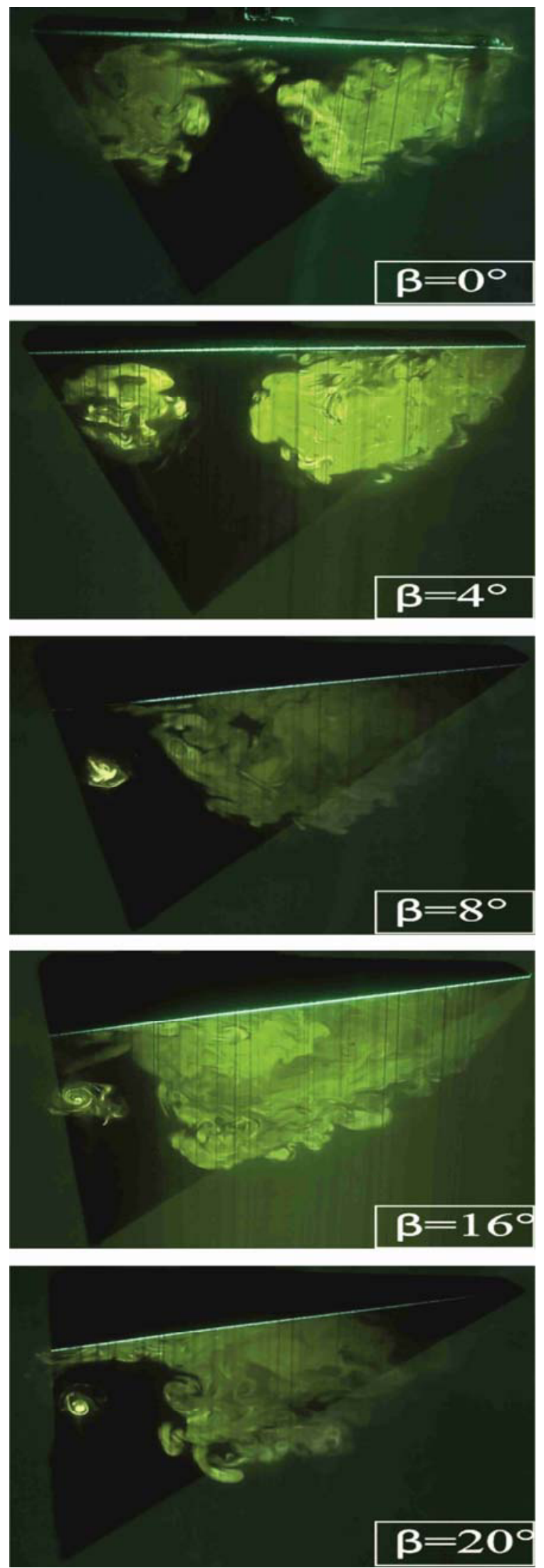

Fig. 7. Vortex formation in end-view plane as a function of yaw angle, $\beta$ at angle of attack, $\alpha=35^{\circ}$. The laser sheet is located at $\mathrm{x} / \mathrm{c}=1.0$ 
It is also reported in the review of this text, for example, taking the dimensionless cord length as $\mathrm{X} / \mathrm{C}=1.0$ and yaw angle, $\beta=0$ there is a symmetrical flow structures in the observed vertical plane crossing the free-stream flow direction as seen in Fig. 8. These flow patterns are demonstrated by dye observations, animations of instantaneous flow data as well as timeaveraged flow data.
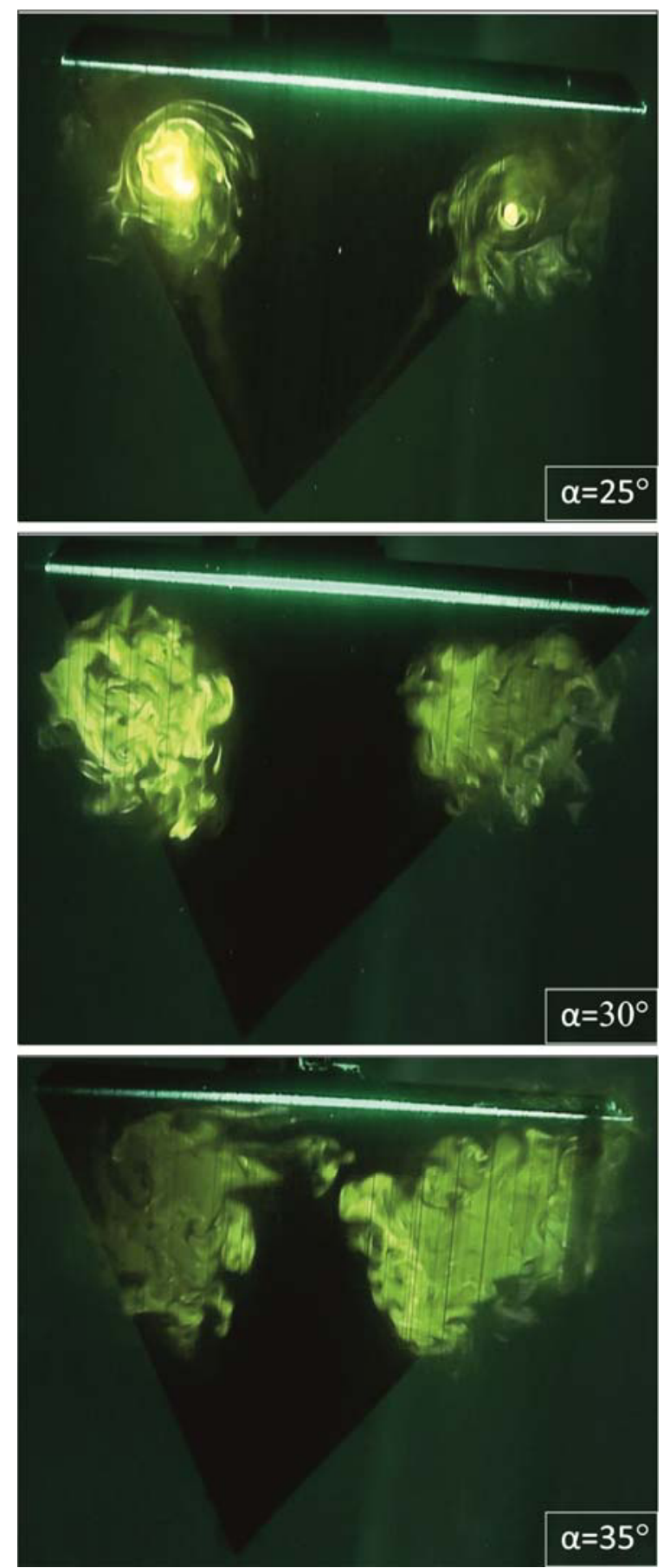

Fig. 8. Vortex formation in end-view plane at angle of attack $\alpha=25^{\circ}, 30^{\circ}$ and $35^{\circ}$ and yaw angle, $\beta=0$. The laser sheet is located at $\mathrm{x} / \mathrm{c}=1.0$
Patterns of time-averaged streamline, $\langle\Psi\rangle$, for angles of attack of $\alpha=25^{\circ} 30^{\circ}$ and $35^{\circ}$ and varying yaw angles, $\beta$ over the range of $0^{\circ} \leq \beta \leq 20^{\circ}$ are present in Figs. 911. The laser sheet is located at a location of $\mathrm{x} / \mathrm{c}=1.0$. Inspection of these images in figures shows that center of well-formed foci, $F_{1}$ and $F_{2}$, saddle points; $S_{1}$ and $S_{2}$ distinctly designate symmetrical flow structures and the domain of main rotating vorticity concentrations. Central points of swirl patterns of streamlines, $F_{1}$ and $F_{2}$ move to the left hand side of wing because of rising yaw angle, $\beta$ from $0^{\circ}$ to $20^{\circ}$. It is observed that the swirling patterns of streamlines, $\langle\Psi>$ indicating flow circulations regions are not symmetrical. Actually, saddle points $S_{1}$ and $\mathrm{S}_{2}$ take place on a boundary. This borderline identifies the border between weak flow region and freestream flow region. Flow structures is extremely susceptible about alteration of yaw angle, $\beta$ and angles of attack, $\alpha$. Well-defined foci, $F_{1}$ and $F_{2}$, are presented by contour of streamlines, $\left\langle\Psi>\right.$. Below foci, $F_{1}$ and $F_{2}$, two saddle points, $\mathrm{S}_{1}$ and $\mathrm{S}_{2}$ are developed.

Patterns of velocity vectors, $<\mathrm{V}>$ clearly show locations of swirling of flow which are coincident with the foci of streamline patterns, $F_{1}$ and $F_{2}$. As seen from Figs. 12-14, distribution of velocity vectors, $\langle\mathrm{V}\rangle$ reveals that as the yaw angle $\beta$, increases, one of the leading edge vortices moves to the left hand side of delta wing and the other leading edge vortex also moves to the left hand side as well close to the center line of delta wing. To study patterns of time-averaged flow data, for angles of attack of $\alpha=25^{\circ}, 30^{\circ}, 35^{\circ}$ and five different values of yaw angle $\beta$, a laser sheet is located at $\mathrm{X} / \mathrm{C}=1.0$ throughout the experiment for PIV measurements. The distribution of time-averaged velocity vectors, $<\mathrm{V}\rangle$ exhibits a localized swirl flow patterns with a high velocity magnitude around the centers of foci, F1 and F2 developing two identifiable recirculation cells. When angles of attack, $\alpha$ is set as $\alpha=25^{\circ}, 30^{\circ}$ and $35^{\circ}$ keeping yaw angle, $\beta$ at $0^{\circ}$ value, it is seen in all cases that the domain and the magnitude of time-averaged velocity vectors, $\langle\mathrm{V}\rangle$ are symmetrical as seen in the first images of Figs. 12-14. But, the magnitude of time-averaged velocity vectors is gradually attenuated as angle of attack, $\alpha$ is increased. As also seen in the dye visualization experiment, when yaw angle is set to $\beta=0^{\circ}$, there are symmetrical flow structures on both sides of central cord axis of the delta wing. But, the magnitude of velocity vectors, $<\mathrm{V}>$ are higher and size of recirculation flow field is larger in the leeward side for higher angles of attack, $\alpha$ and higher yaw angles, $\beta$ at $\mathrm{X} / \mathrm{C}=1.0$. Both central points of foci, F1 and F2 move towards the leeward side of the wing. 

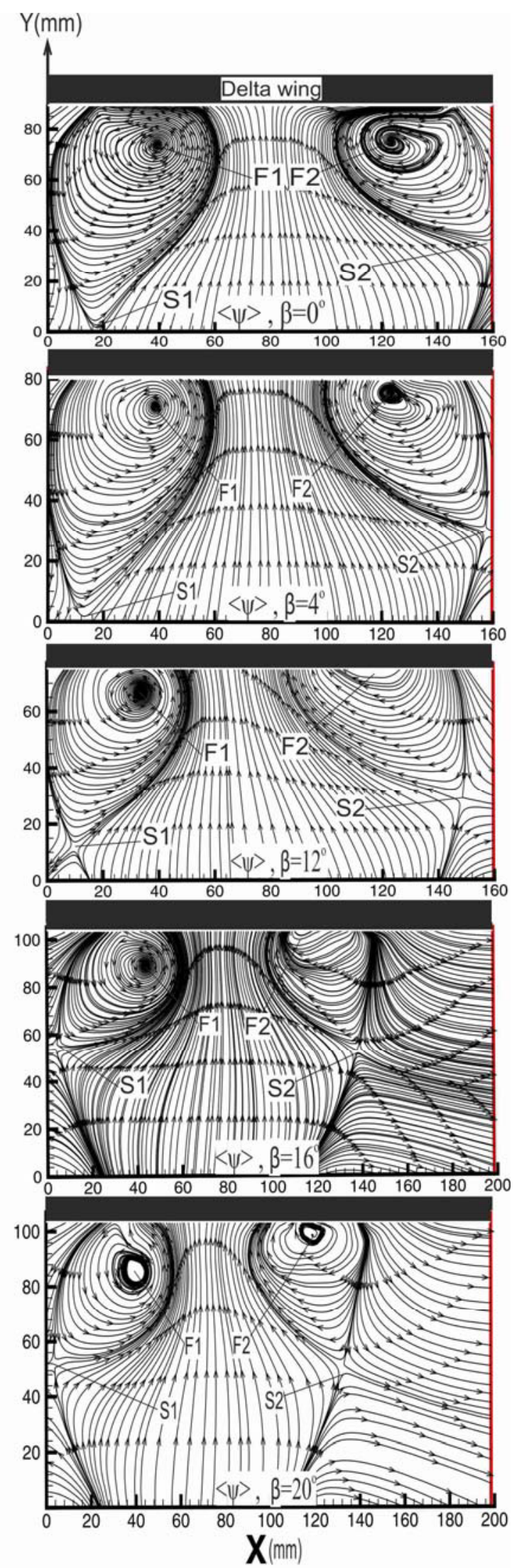

Fig. 9. Patterns of time-averaged streamlines, $\langle\Psi\rangle$, with variation of yaw angle, $\beta$ for angle of attack, $\alpha=25^{\circ}$. The laser sheet is located at $\mathrm{X} / \mathrm{c}=1.0$
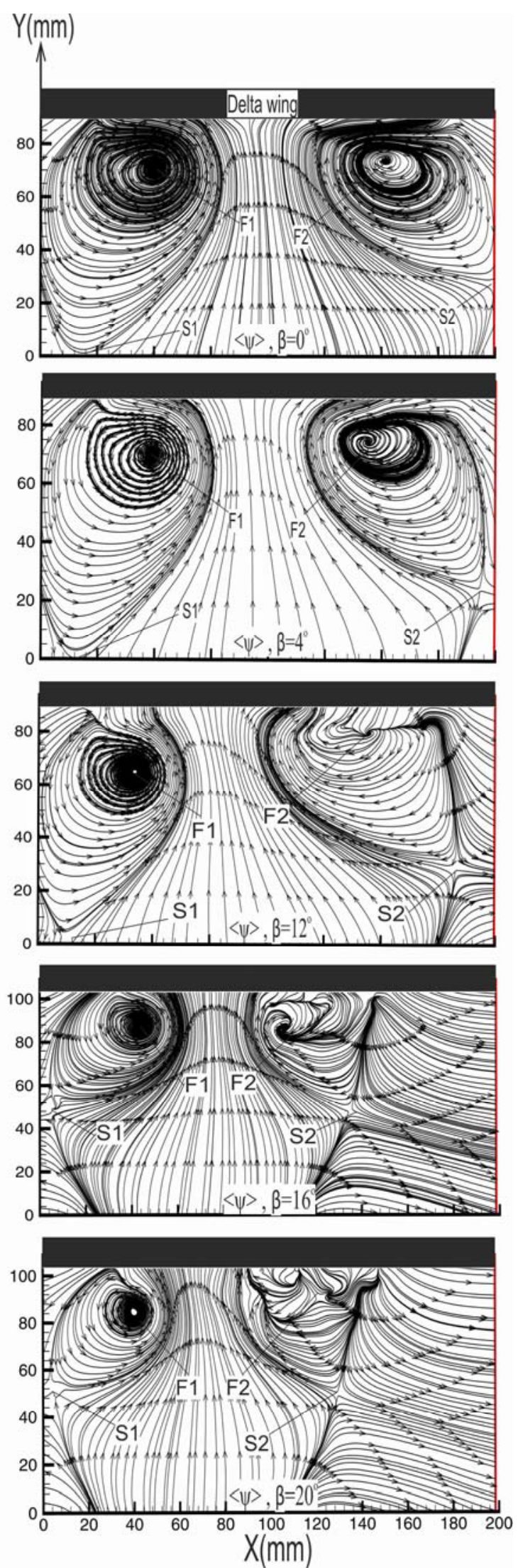

Fig. 10. Patterns of time-averaged streamline, $\langle\Psi\rangle$, with variation of yaw angle, $\beta$ for angle of attack, $\alpha=30^{\circ}$. The laser sheet is located at $\mathrm{X} / \mathrm{c}=1.0$ 

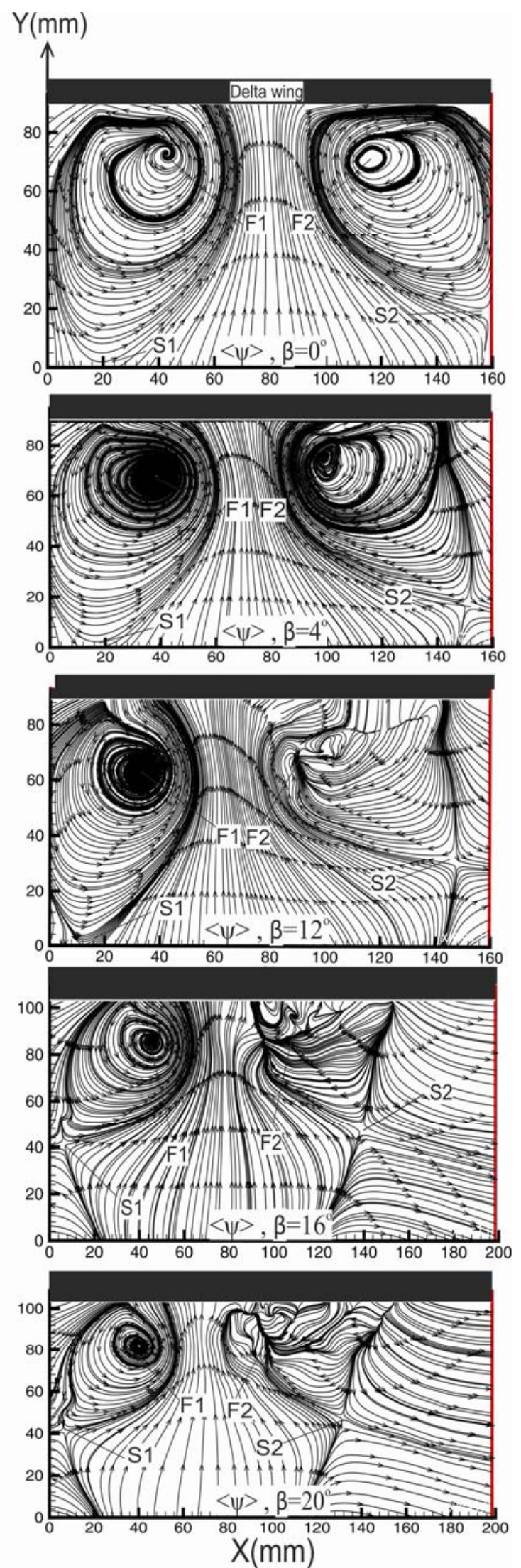

Fig. 11. Patterns of time-averaged streamline, $\langle\Psi\rangle$, with variation of yaw angle, $\beta$ for angle of attack $\alpha=35^{\circ}$. The laser sheet is located at $\mathrm{X} / \mathrm{c}=1.0$
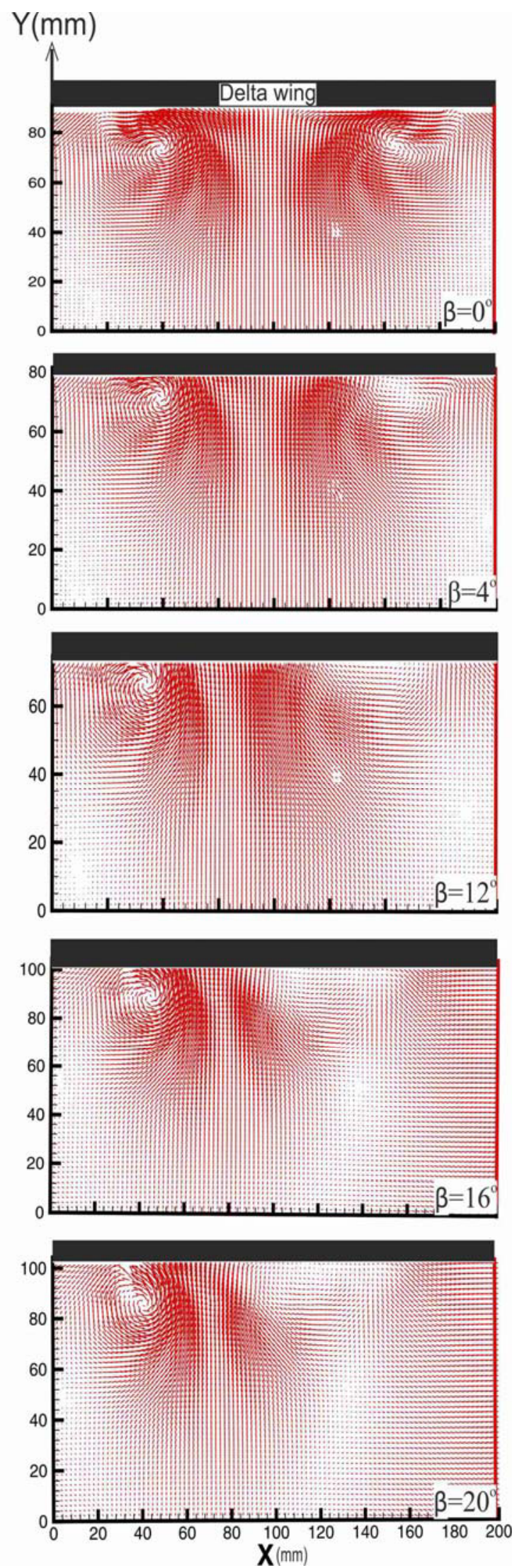

Fig. 12. Patterns of time-averaged distribution of velocity vectors, $\langle\mathrm{V}>$ with variation of yaw angle, $\beta$ for angle of attack, $\alpha=25^{\circ}$. The laser sheet is located at $\mathrm{x} / \mathrm{c}=1.0$. 

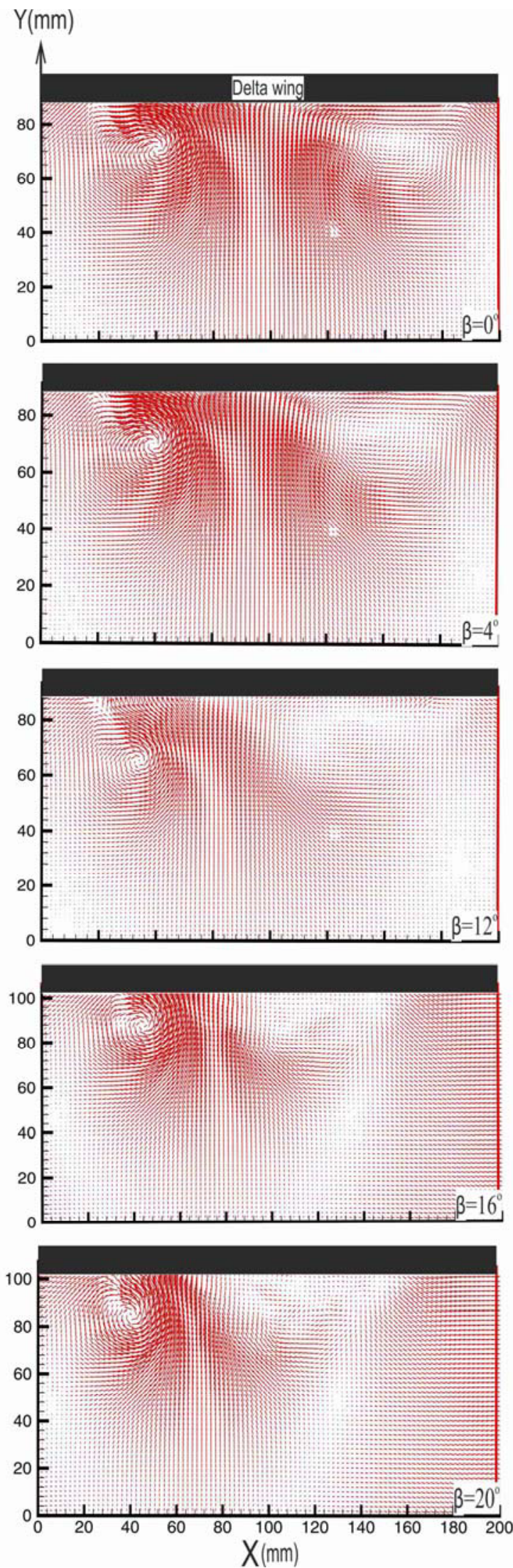

Fig. 13. Patterns of time-averaged distribution of velocity vectors, $\langle\mathrm{V}\rangle$ with variation of yaw angle, $\beta$ for angle of attack, $\alpha=30^{\circ}$. The laser sheet is located at $\mathrm{x} / \mathrm{c}=1.0$
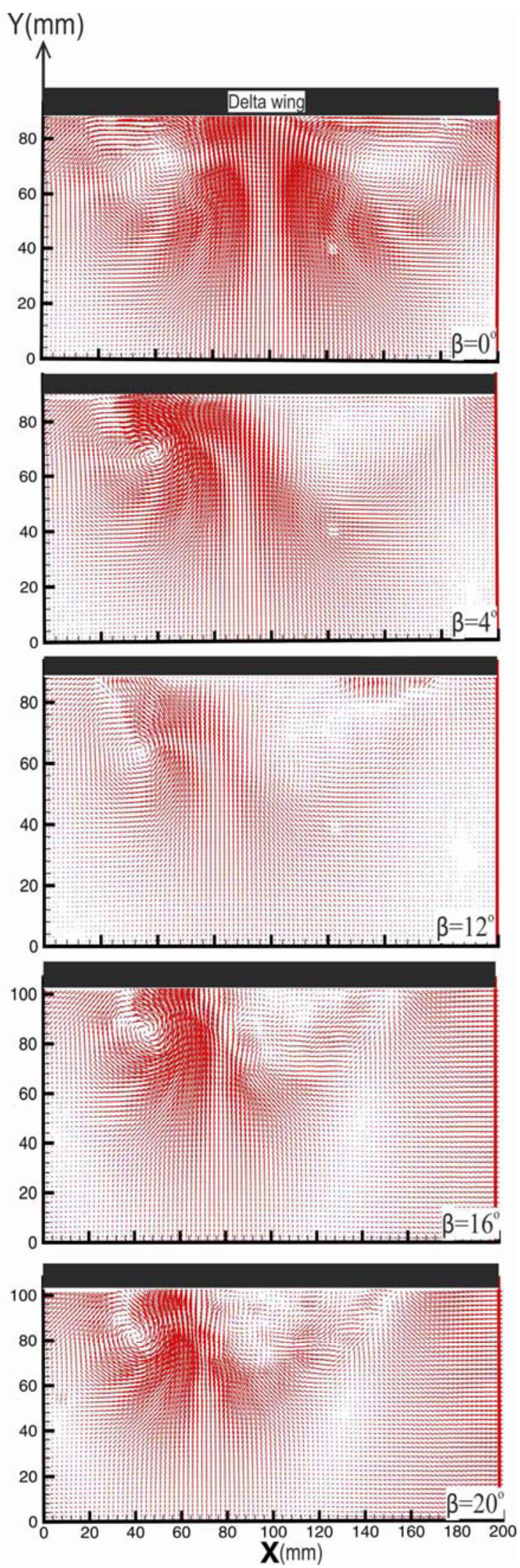

Fig. 14. Patterns of time-averaged distribution of velocity vectors, $<\mathrm{V}>$ with variation of yaw angle, $\beta$ for angle of attack, $\alpha=35^{\circ}$. The laser sheet is located at $\mathrm{x} / \mathrm{c}=1.0$ 


\section{Conclusion}

In this work, basic features of counter rotating leading edge vortices in end-view planes of the delta wing with $70^{\circ}$ sweep angle, $\Lambda$ were studied both qualitatively and quantitatively using Rhodamine dye and the PIV system. Experiments were conducted by altering angles of attack over the range of $25^{\circ} \leq \alpha \leq 35^{\circ}$ and yaw angles, $\beta$ over the range of $0^{\circ} \leq \beta \leq 20^{\circ}$ for the present study. Rhodamine dye visualizations were performed in end-view planes passing through rear end of delta wing at $\mathrm{X} / \mathrm{C}=1.0$ in order to observe alterations of vortical flow structures under the effect of yaw angles, $\beta$.

In end-view plane, symmetrical flow structures are developed in the case of zero yaw angles, $\beta$ for all case of angles of attack, $\alpha$. In the light of present experiments it is seen that with increasing yaw angle, $\beta$ symmetrical flow structure is disrupted continuously.

The streamline topology $\langle\Psi\rangle$ shows that there are two saddle points, $S_{1}$ and $S_{2}$, and foci, $F_{1}$ and $F_{2}$. Saddle points, $S_{1}$ and $S_{2}$, are clearly seen and located below the foci, $F_{1}$ and $F_{2}$. The streamline patterns, $\langle\Psi\rangle$ show a well-defined swirl pattern. The saddle points, $\mathrm{S}_{1}$ and $\mathrm{S}_{2}$, and center of foci, $F_{1}$ and $F_{2}$, gradually move downward and get closer to each other as angle of attack, $\alpha$ is increased because of stall flow regions expends in size as a function of both angle of attack, $\alpha$ and yaw angle, $\beta$. Patterns of streamlines, $\langle\Psi\rangle$ of counter rotating flow recirculation are dissimilar in terms of size and magnitude. Differences between size and severity of vortices are easily seen in PIV results that are quantitatively visualized in terms of streamlines, $\langle\Psi\rangle$ and velocity vectors, $<\mathrm{V}>$.

Time-averaged velocity vectors, $<\mathrm{V}>$ indicates a well-defined a pair of identical swirling flow cells for the case of zero yaw angle, $\beta$ for all cases of angles of attack, that are considered in the present work. But, a well-defined recirculating flow region on the windward side gradually attenuates and diminishes and finally a single flow circulating loop is only seen in the image of velocity vector distributions $\langle\mathrm{V}\rangle$ on the leeward side in the case of higher yaw angle, $\beta \geq 12^{0}$. Increasing yaw angle, $\beta$ from $\beta=0^{\circ}$ to $\beta=4^{\circ}$ flow structures are subjected to small changes. Symmetrical flow structures are dramatically altered at a yaw angle of $\beta=20^{\circ}$ compared with the case of yaw angles from $\beta=0^{0}$ to $\beta=4^{0}$. The distribution of time-averaged velocity vectors, $<\mathrm{V}>$ exhibits several small scales circulations presented by localized swirl patterns of streamlines.

\section{Acknowledgments}

This study was supported by The Scientific and Technological Research Council of Turkey (TÜBİTAK) under contract number of 114M497 and Çukurova University Scientific Research Unit under contract number of FLY-2016-5845.

\section{References}

1. Akilli, H., Sahin, B., and Rockwell, D., (2001), "Control of Vortex Breakdown by a TransverselyOriented Wire", Physics of Fluids, 13(2):452-463

2. Anderson, J.D., (201), "Fundamentals of aerodynamics", McGraw-Hill Higher Education, ISBN 0-07-118146-6

3. Canpolat C., Yayla S., and Sahin B., (2012), "Observation of the Vortical Flow over a Yawed Delta Wing, Journal of Aerospace Engineering",25(4):613-626 4. Canpolat, C., Yayla, S., Sahin, B., and Akilli, H., (2009), "Dye Visualization of the Flow Structure over a Yawed Nonslender Delta Wing", The Journal of Aircraft, 46(5), pp. 1818-1822

5. Karasu I., Sahin B., Akilli H., and Canpolat C., (2015) "Dye Visualization of a Yawed Slender Delta Wing", Journal of Thermal Engineering, Yildiz Technical University Press, Istanbul, Turkey, 1(2-7): 646-654

6. Lambourne N. C., and Bryer D. W., (1961), "The Bursting of Leading Edge Vortices--Some Observations and Discussion of the Phenomenon", Aeronautical Research Council Reports And Memoranda, Reports and Memoranda No. 3282

7. Meinhart and Soloff, (1999), "Cleanvec software", Turbulence Laboratory, University of Illinois at UrbanaChampaign

7. Ozgoren, M., Sahin, B., and Rockwell, D., (2002), "Vortex Structure on a Delta Wing at High Angle-ofAttack", AIAA Journal, 40(2):285- 292

9. Sarpkaya, T., (1971), "On stationary and travelling vortex breakdowns", Journal of Fluid Mechanics 45(03):545-559

10. Sohn, M. H., Lee, K. Y., and Chang, J. W., (2004), "Vortex Flow Visualization of a Yawed Delta Wing with Leading-Edge Extension”, Journal of Aircraft, 41(2)

11. Sohn, M. H., and Chang J. W., (2010), "Effect of a centerbody on the vortex flow of a double-delta wing with leading edge extension", Aerospace Science and Technology 14(1):11-18

12. Yaniktepe, B. and Rockwell D., (2005), "Flow Structure on Diamond and Lambda Planforms: TrailingEdge Region”, AIAA Journal, 43(7), pp. 1490-1500

13. Yayla, S., Canpolat, C. Sahin, B., and Akilli, H., 82010), "Yaw angle effect on flow structure over the nonslender diamond wing", AIAA journal, 48(10):24572461

14. Yayla, S., Canpolat, C., Sahin, B., and Akilli, H., (2010), "Effect of Yaw Angle on the Formation of Vortex Breakdown over the Diamond Wing", Journal of Thermal Science and Technology, 30(1): 79-89 